\title{
Cyanoacrylate glue in the management of blue rubber bleb nevus syndrome
}

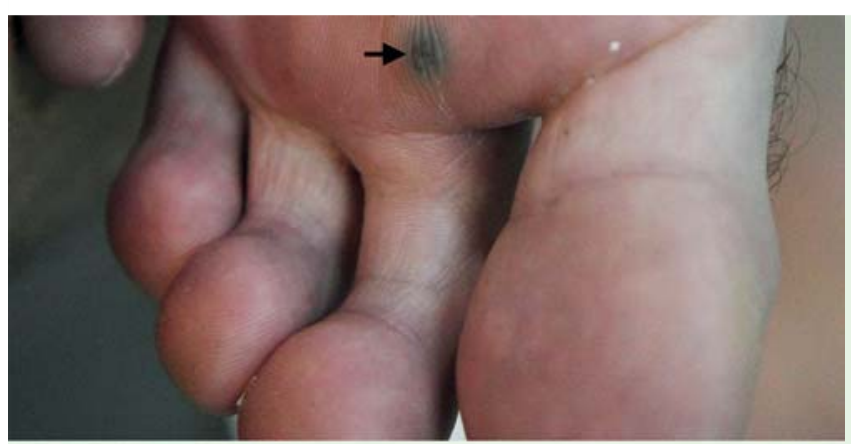

Fig. 1 Case 1. Hemangiomas of the foot.
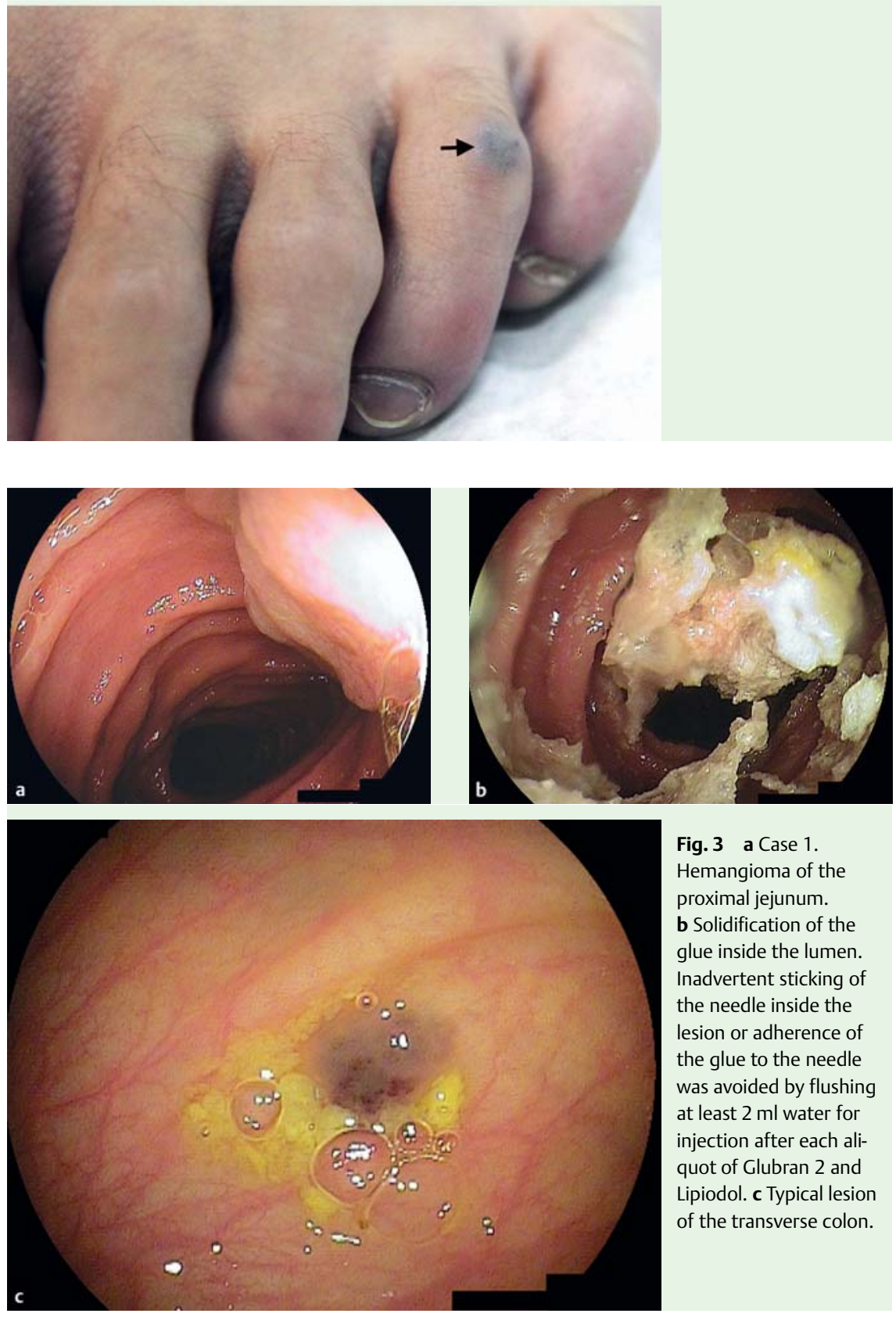

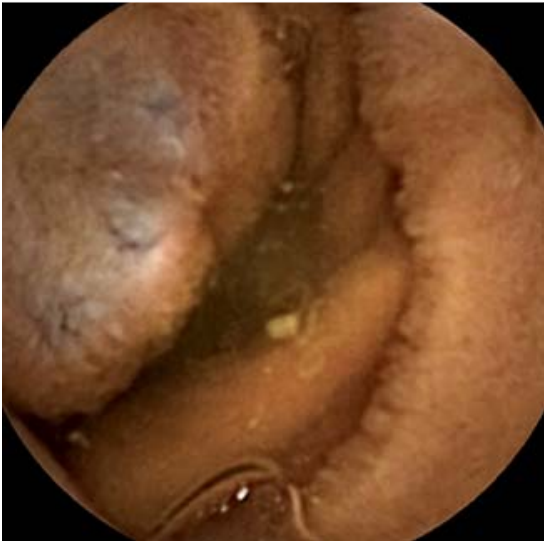

Fig. 2 Case 1. Capsule endoscopy picture of a bluish, nipple-like rubbery vascular lesion of the jejunum, compatible with a blue rubber bleb nevus.

Case 1. A 16-year-old boy with blue rubber bleb nevus syndrome (BRBNS) was admitted for occult bleeding. He had a history of gastric and colonic hemangiomas treated 2 years earlier by argon plasma coagulation and alcohol injection respectively. Clinical examination showed a few hemangiomas on his back and feet (๑ Fig. 1).

Capsule endoscopy (Pillcam SB2; Given Imaging, Yoqneam, Israel) disclosed three blebs of the small bowel ( Fig. 2), and the patient underwent double-balloon enteroscopy (Fujinon, Saitama, Japan).

A 2-cm bleb was found at the jejunum, two smaller lesions at the ileum, and one at the transverse colon. N-butyl-2cyanoacrylate and methacryloxysulfolane (Glubran 2), $1 \mathrm{ml}$, diluted with Lipiodol, $1 \mathrm{ml}$, was injected in aliquots of $1 \mathrm{ml}$ per injection and up to $4 \mathrm{ml}$ at each lesion ( Fig. 3). No complications occurred. At 6-month follow-up the patient had no anemia.

Case 2. An 11-year-old girl with BRBNS was addressed for hematochezia due to colonic blebs. Colonoscopy revealed a bleeding lesion at the cecum ( $\bullet$ Fig. 4).

After initial failure to stop the bleeding with a hemostatic grasper (Coagrasper, Olympus), Glubran 2 was injected successfully. Two more blebs of the right colon were treated similarly. After 2 years the patient remained asymptomatic. 


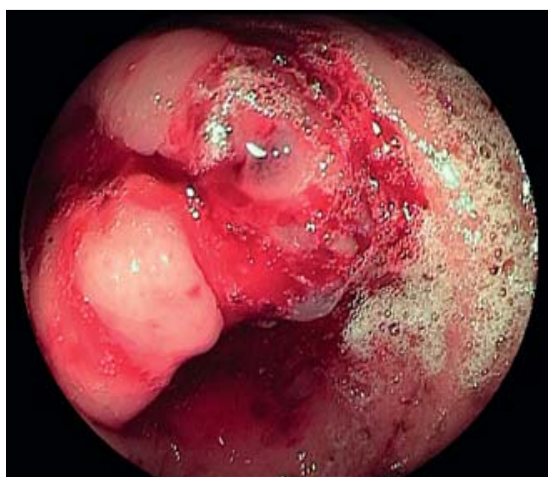

Fig. 4 Case 2. Bleeding hemangioma of the cecum near the ileocecal valve.

BRBNS is a rare condition characterized by multiorgan cavernous hemangiomas. Gastrointestinal bleeding is the major problem, and several endoscopic therapeutic approaches have been proposed: Nd:YAG laser, bipolar or argon plasma coagulation, band ligation, snare resection, and sclerotherapy [1-4]. Our patients were treated successfully by Glubran 2 injection, commonly used in the management of gastric varices. Endoscopic obliteration of cavernous hemangiomas with n-butyl-2-cyanoacrylate (Histoacryl) has been previously described, in the rectum [5]. However, these appear to be the first cases of cyanoacrylate glue injection to control bleeding in the small bowel and colon in the setting of BRBNS.

Endoscopy_UCTN_Code_TTT_1AO_2AD

Competing interests: None

G. Mavrogenis ${ }^{1}$, D. Coumaros ${ }^{1}$,

D. Tzilves ${ }^{1}$, E. Rapti ${ }^{1}$, G. Stefanidis ${ }^{2}$, J. Leroy $^{3}$, F. Becmeur ${ }^{4}$

1 Department of Gastroenterology, University Hospital, Strasbourg, France

2 Department of Gastroenterology, Athens Naval Hospital, Athens, Greece

3 Department of Digestive Surgery, University Hospital, Strasbourg, France.

4 Department of Pediatric Surgery, University Hospital, Strasbourg, France

\section{References}

1 Shahed M, Hagenmüller F, Rösch T et al. A 19year-old female with blue rubber bleb nevus syndrome. Endoscopic laser photocoagulation and surgical resection of gastrointestinal angiomata. Endoscopy 1990; 22: 54-56
2 Maunoury V, Turck D, Brunetaud JM et al. Blue rubber bleb nevus syndrome. 3 cases treated with a Nd:YAG laser and bipolar electrocoagulation [in French with English abstract]. Gastroenterol Clin Biol 1990; 14: 593-595

$3 \mathrm{Bak} Y T$, Oh CH, Kim JH. Blue rubber bleb naevus syndrome: endoscopic removal of the gastrointestinal hemangiomas. Gastrointest Endosc 1997; 45: 90-92

4 Arguedas MR, Wilcox CM. At the focal point. Blue rubber bleb nevus syndrome. Gastrointest Endosc 1999; 50: 544

5 Zurakowski J, Swiercz P, Wróblewski T et al. Diffuse cavernous hemangioma of rectosigmoid colon treated with n-butyl-2-cyanoacrylate injections. Endoscopy 2008; 40: E120-121

Bibliography

DOI $10.1055 / \mathrm{s}-0030-1256425$

Endoscopy 2011; 43: E291 - E292

(c) Georg Thieme Verlag KG Stuttgart · New York . ISSN 0013-726X

Corresponding author

\section{Coumaros, MD}

IRCAD/EITS

University Hospital

1 Place de l'Hôpital

67091 Strasbourg

France

Fax: +33-3-88751521

coumarosd@wanadoo.fr 